\title{
LARGE PERIODIC LYAPUNOV EQUATIONS: ALGORITHMS AND APPLICATIONS
}

\author{
Daniel Kressner \\ Institut für Mathematik, MA 4-5 \\ Technische Universität Berlin \\ D-10623 Berlin, Germany. \\ e-mail: kressner@math.tu-berlin.de
}

Keywords: Periodic discrete-time systems, Lyapunov equations, model reduction, Riccati equations

\begin{abstract}
Two algorithms for the solution of discrete-time periodic Lyapunov equations are presented. The first one is a variant of the squared Smith iteration, which is solely based on matrix multiplications and thus attractive to parallel computing environments. The second algorithm is based on Krylov subspaces and employs a recently developed variant of the block Arnoldi algorithm. It is particularly suited for periodic Lyapunov equations with large and sparse coefficient matrices. We also demonstrate how these methods can be applied to balanced truncation model reduction of periodic discrete-time systems and the solution of periodic Riccati equations.
\end{abstract}

\section{Introduction}

A discrete-time periodic Lyapunov equation (or periodic Stein equation) is a system of equations of the form

$$
A^{(l) T} X^{(l+1)} A^{(l)}-X^{(l)}=-W^{(l)}
$$

for $l=1,2, \ldots$, where the coefficients $A^{(l)}, W^{(l)}$ and the solutions $X^{(l)}$ are real $n \times n$ matrices which are periodic with period $p$, i.e., $A^{(l+p)}=A^{(l)}, W^{(l+p)}=W^{(l)}$ and $X^{(l+p)}=$ $X^{(l)}$. Stacking (1) yields the equivalent lifted representation

$$
\mathcal{A}^{T} \mathcal{X} \mathcal{A}-\mathcal{X}=-\mathcal{W}
$$

where

$$
\mathcal{A}=\left[\begin{array}{cccc}
0 & & & A^{(p)} \\
A^{(1)} & \ddots & & \\
& \ddots & \ddots & \\
& & A^{(p-1)} & 0
\end{array}\right]
$$

and $\mathcal{X}, \mathcal{W}$ are $p n \times p n$ block diagonal matrices with diagonal entries $X^{(1)}, \ldots, X^{(p)}$ and $W^{(1)}, \ldots, W^{(p)}$, respectively.

Such equations appear in solving periodic state-feedback problems and computing gradients for optimal periodic output feedback problems [21, 24]. Further applications related to discrete-time periodic systems include the solution of Riccati equations [4], minimal realisations [22] and model reduction $[15,23]$.
It is well known that (2) has a unique solution if and only if $\mathcal{A}$ has no reciprocal eigenvalues. This equalises the condition that the product $A^{(k)} A^{(k-1)} \cdots A^{(1)}$ has no reciprocal eigenvalues. Furthermore, it was shown in [6] that the solution $\mathcal{X}$ of (2) is block diagonal, this implies that the diagonal blocks of $\mathcal{X}$ represent the solution of (1).

In this paper, we consider periodic Lyapunov equations with large and sparse coefficient matrices $A^{(1)}, \ldots, A^{(p)}$. In this case, all known solution algorithms [7, 19, 21] fail to solve (1) due to excessively high memory and computing time requirements.

We show two ways to remedy this situation. The first one is presented in Section 2 and is an extension of the squared Smith Iteration which has already been successfully applied to the case $p=1$ [3]. Although the whole method is based on matrixby-matrix multiplications, and thus very attractive to parallel computing environments, it does not help very much reduce memory requirements. Consequently, the problem sizes that can be tackled by this method are still very limited. In Section 3 we therefore present the second way, a Krylov subspace based method. If all coefficient matrices are sparse this method has the potential to reduce memory and time requirements to a tiny fraction. Again, such a method has already been implemented for the case $p=1$, see e.g. [12]. Sections 4 and 5 demonstrate how the newly developed methods can be applied to balanced truncation model reduction and the solution of periodic Riccati equations.

\section{The Squared Smith Iteration}

The Lyapunov equation (2) can be formulated in fix point form $\mathcal{X}=\mathcal{A}^{T} \mathcal{X} \mathcal{A}+\mathcal{W}$, from which the fix point iteration

$$
\mathcal{X}_{0}:=\mathcal{W}, \quad \mathcal{X}_{k+1}:=\mathcal{A}^{T} \mathcal{X}_{k} \mathcal{A}+\mathcal{W}, \quad k=0,1, \ldots,
$$

can be derived. The convergence of this iteration to $\mathcal{X}$ is guaranteed if $\rho(\mathcal{A})<1$, which is equivalent to $\rho\left(A^{(p)} A^{(p-1)} \cdots A^{(1)}\right)<1$. The convergence rate is linear, a quadratically convergent version was suggested in [17]. This so called squared Smith iteration is obtained by setting $\mathcal{A}_{0}:=\mathcal{A}, \mathcal{X}_{0}:=\mathcal{W}$ and considering

$$
\mathcal{X}_{k+1}:=\mathcal{A}_{k}^{T} \mathcal{X}_{k} \mathcal{A}_{k}+\mathcal{X}_{k}, \mathcal{A}_{k+1}=\mathcal{A}_{k}^{2}, \quad k=0,1, \ldots
$$

If we want to boil this equation down to the matrix coefficient level we need to deal with the seemingly wild reordering of 
the coefficients $A_{k}^{(l)}$ and $X_{k}^{(l)}$. A helpful tool is provided by permutation function and matrices.

Definition 1 Let $\sigma:\{1, \ldots, p\} \rightarrow\{1, \ldots, p\}$ be a permutation, then $\mathcal{P}(\sigma)$ is the $p n \times$ pn block permutation matrix with $n \times n$ block entries

$$
\mathcal{P}_{i j}(\sigma):=\left\{\begin{aligned}
I_{n} & \text { if } \sigma(i)=j, \\
0 & \text { otherwise, }
\end{aligned}\right.
$$

where $i, j=1, \ldots, p$ and $I_{n}$ is the $n \times n$ identity matrix.

The few facts we need to know about permutations are summarised in the following lemma, where $\operatorname{diag}\left(D^{(1)}, \ldots, D^{(p)}\right)$ denotes the $p n \times p n$ block diagonal matrix with diagonal entries $D^{(1)}, \ldots, D^{(p)}$.

\section{Lemma 2}

1. $\mathcal{P}(\sigma)^{T}=\mathcal{P}\left(\sigma^{-1}\right), P(\sigma \circ \sigma)=P(\sigma)^{2}$.

2. Let $\sigma_{0}(1):=p, \sigma_{0}(2)=1, \ldots, \sigma_{0}(p)=p-1$, then

$$
P\left(\sigma_{0}\right)^{T} \mathcal{A}=\operatorname{diag}\left(A^{(1)}, \ldots, A^{(p)}\right) .
$$

3. Let $\mathcal{D}=\operatorname{diag}\left(D^{(1)}, \ldots, D^{(p)}\right)$, then

$$
\mathcal{P}(\sigma)^{T} \mathcal{D} \mathcal{P}(\sigma)=\operatorname{diag}\left(D^{\sigma^{-1}(1)}, \ldots, D^{\sigma^{-1}(p)}\right) .
$$

Proof. By direct computation.

Now we can rewrite (4) so that each factor is in block diagonal form:

$$
\begin{gathered}
\mathcal{X}_{k+1}:=\left(P\left(\sigma_{k}\right) \mathcal{A}_{k}\right)^{T}\left(P\left(\sigma_{k}\right)^{T} \mathcal{X}_{k} P\left(\sigma_{k}\right)\right)\left(P\left(\sigma_{k}\right) \mathcal{A}_{k}\right)+\mathcal{X}_{k} \\
\mathcal{A}_{k+1}:=\mathcal{A}_{k}^{2}, \quad \sigma_{k+1}:=\sigma_{k} \circ \sigma_{k},
\end{gathered}
$$

where $\sigma_{0}$ is defined as in Lemma 2 . This not only proves that the iterates $\mathcal{X}_{k+1}$ stay in block diagonal form it also enables us to rewrite (4) in terms of the coefficient matrices:

$$
\begin{aligned}
X_{0}^{(l)} & :=W^{(l)}, \\
A_{0}^{(l)} & :=A^{(l)}, \\
X_{k+1}^{(l)} & :=A_{k}^{(l) T} X_{k}^{\sigma_{k}^{-1}(l)} A_{k}^{(l)}+X_{k}^{(l)}, \\
A_{k+1}^{(l)} & :=A_{k}^{\sigma^{-1}(l)} A_{k}^{(l)}, \\
\sigma_{k+1} & :=\sigma_{k} \circ \sigma_{k}, \quad l=1, \ldots, p .
\end{aligned}
$$

Let $\rho:=\rho\left(A^{(p)} A^{(p-1)} \cdots A^{(1)}\right)<1$, then the convergence theory of squared Smith iterations [17] applied to (4) shows that for any $\epsilon>0$ there is a constant $C_{\epsilon}>0$ so that

$$
\left\|X^{(l)}-X_{k}^{(l)}\right\|_{2} \leq C_{\epsilon}(\rho-\epsilon)^{2^{k} / p} \cdot \max _{l^{\prime} \in\{1, \ldots, p\}}\left\|W^{\left(l^{\prime}\right)}\right\|_{2} .
$$

If the right-hand side matrix $\mathcal{W}$ in (2) is given in factored form, $\mathcal{W}=\mathcal{B B}^{T}$, then $\mathcal{W}$ is symmetric and positive semidefinite. The same holds for the solution matrix $\mathcal{X}$ provided that $\rho<1$, see e.g. [9]. Furthermore, if $\mathcal{B}$ has low rank, then $\mathcal{X}$ stands good chances that its numerical rank is also comparably low [1]. We can now use the following modification of the iteration (5) to reduce its memory requirements and enhance its numerical accuracy. Let $\mathcal{B}=\operatorname{diag}\left(B^{(1)}, \ldots, B^{(p)}\right)$, where $B^{(l)}$ is an $n \times m_{l}$ matrix with $m_{l}=\operatorname{rank}\left(B^{(l)}\right) \leq n$, then

$$
\begin{aligned}
L_{0}^{(l)} & :=B^{(l)}, \\
A_{0}^{(l)} & :=A^{(l)} \\
\hat{L}_{k+1}^{(l)} & :=\left[L_{k}^{\sigma_{k}^{-1}(l)}, A_{k}^{(l) T} L_{k}^{\sigma_{k}^{-1}(l)}\right], \\
A_{k+1}^{(l)} & :=A_{k}^{\sigma_{k}^{-1}(l)} A_{k}^{(l)}, \\
\sigma_{k+1} & :=\sigma_{k} \circ \sigma_{k}, \quad l=1, \ldots, p .
\end{aligned}
$$

If we set $L_{k+1}^{(l)}=\hat{L}_{k+1}^{(l)}$ in (7) then its number of columns would double in each iteration. Instead, we propose to compute a rank-revealing $L Q$-factorization [10]

$$
\hat{L}_{k+1}^{(l)}=\left[\begin{array}{ll}
L_{k+1}^{(l)} & 0
\end{array}\right] Q_{k+1}^{(l)},
$$

where $Q_{k+1}^{(l)}$ is an orthonormal matrix and $\operatorname{rank}\left(\hat{L}_{k+1}^{(l)}\right)=$ $\operatorname{rank}\left(L_{k+1}^{(l)}\right)$. As the iteration proceeds $L_{k}^{(l)} L_{k}^{(l) T}$ converges to $X_{k}^{(l)}$ with the rate given by (6). It is pointed out in [3] that this approach could also be used to compute a low rank approximation of $X_{k}^{(l)}$, either by increasing the tolerance threshold for determining the numerical rank of $\hat{L}_{k+1}^{(l)}$ or by bounding the maximal number of columns in $L_{k+1}^{(l)}$.

The iteration (5) is solely based on matrix multiplications making it attractive to parallel computing environments. Existent algorithms for solving periodic Lyapunov equations are based on periodic Schur and Hessenberg decompositions which - as the standard Schur and Hessenberg decompositions - do not easily yield parallelisable algorithms for their computation. Thus, it can be expected that the iterations (5) or (7) greatly outperform existent algorithms when the solution of the periodic Lyapunov equation (1) is computed on a parallel machine. This has been confirmed for the case $p=1$ [3].

It must be said, however, that the need for saving the coefficient matrices of $\mathcal{A}^{2^{k}}$ makes squared Smith iterations impracticable when the coefficient matrices of $\mathcal{A}$ are large and sparse or only implicitly, through their action on a vector $x$, defined. In the next section we therefore present an alternative way for computing an approximate solution of (1).

\section{A Krylov Subspace Method}

In the following we assume that each right hand side of the periodic Lyapunov equation (1) is factored into $W^{(l)}=$ $B^{(l)} B^{(l) T}$, where $B^{(l)} \in \mathbb{R}^{n \times m_{l}}$ has full rank. Let $\mathcal{B}=$ $\operatorname{diag}\left(B^{(1)}, \ldots, B^{(p)}\right)$, then

$$
\mathcal{K}_{k}(\mathcal{A}, \mathcal{B})=\operatorname{span}\left(\mathcal{B}, \mathcal{A B}, \ldots, \mathcal{A}^{k-1} \mathcal{B}\right)
$$

is called a block Krylov subspace of order $k$. Krylov subspace based methods for the standard Lyapunov equation (2), see 
e.g. [12], first compute the solution $\mathcal{Y}$ of the reduced Lyapunov equation

$$
\left(\mathcal{U}_{k}^{T} \mathcal{A} \mathcal{U}_{k}\right)^{T} \mathcal{Y}\left(\mathcal{U}_{k}^{T} \mathcal{A} \mathcal{U}_{k}\right)-\mathcal{Y}=-\left(\mathcal{U}_{k}^{T} \mathcal{B}\right)\left(\mathcal{U}_{k}^{T} \mathcal{B}\right)^{T}
$$

where $\mathcal{U}_{k}$ is an orthonormal basis of $\mathcal{K}_{k}(\mathcal{A}, \mathcal{B})$. Then, for sufficiently large $k$, the matrix $\mathcal{U}_{k} \mathcal{Y} \mathcal{U}_{k}^{T}$ is a good approximation of $\mathcal{X}$, the solution of the unreduced Lyapunov equation (2). In the case of periodic Lyapunov equations it is crucial to choose $\mathcal{U}_{k}$ in block diagonal form so that the structures of $\mathcal{A}$ and $\mathcal{B}$ carry over to $\mathcal{U}_{k}^{T} \mathcal{A} \mathcal{U}_{k}$ and $\mathcal{U}_{k}^{T} \mathcal{B}$.

A block Arnoldi decomposition of order $k$ has the form

$$
\mathcal{A} \mathcal{U}_{k}=\mathcal{U}_{k+1} \hat{\mathcal{H}}_{k}
$$

where the orthonormal columns of the matrix $\mathcal{U}_{k+1}$ contain those of $\mathcal{U}_{k}$. It is well known that under certain conditions on $\hat{\mathcal{H}}_{k}$ the existence of such a decomposition implies that $\mathcal{U}_{k}$ is the basis of a block Krylov subspace. We now give a brief outline of a block Arnoldi-like algorithm that computes a decomposition of the form (9), where $\mathcal{U}_{k}$ is a block diagonal matrix. A detailed analysis of this algorithm and its implementation issues are currently under investigation and will be published in a forthcoming paper.

\section{Algorithm 3}

Input: Matrices $A^{(l)} \in \mathbb{R}^{n \times n}, B^{(l)} \in \mathbb{R}^{n \times m_{l}}$, $l=1, \ldots, p$, and an integer $k \leq n$.

Output: Matrices $U_{[k]}^{(l)}, U_{[k+1]}^{(l)}, \hat{H}_{k}^{(l)}, l=1, \ldots, p$, so that

$$
\begin{aligned}
\mathcal{U}_{k} & =\operatorname{diag}\left(U_{[k]}^{(1)}, \ldots, U_{[k]}^{(p)}\right), \\
\mathcal{U}_{k+1} & =\operatorname{diag}\left(U_{[k+1]}^{(1)}, \ldots, U_{[k+1]}^{(p)}\right), \\
\hat{\mathcal{H}}_{k}= & {\left[\begin{array}{cccc}
0 & & & \hat{H}_{k}^{(p)} \\
\hat{H}_{k}^{(1)} & \ddots & & \\
& \ddots & \ddots & \\
& & \hat{H}_{k}^{(p-1)} & 0
\end{array}\right] }
\end{aligned}
$$

satisfy a block Arnoldi decomposition (9).

$$
\begin{gathered}
\text { FOR } l=1, \ldots, p \\
\text { Compute a } Q R \text { decol } \\
U_{[1]}^{(l)}=U_{1}^{(l)} \\
\text { END FOR } \\
\text { FOR } k=1,2, \ldots \\
\text { FOR } l=1, \ldots, p \\
\text { IF } l=1 \text { THEN } \\
l^{\prime}=p
\end{gathered}
$$$$
\text { Compute a } Q R \text { decomposition } B^{(l)}=U_{1}^{(l)} R^{(l)} \text {. }
$$

$$
l^{\prime}=l-1
$$

END IF

$F_{k}^{(l)}=U_{[k]}^{(l) T} A^{\left(l^{\prime}\right)} U_{k}^{\left(l^{\prime}\right)}$

$V=A^{\left(l^{\prime}\right)} U_{k}^{\left(l^{\prime}\right)}-U_{[k]}^{(l)} F_{k}^{(l)}$

Compute a rank-revealing $Q R$ decomposition:

$$
\begin{gathered}
V P=U_{k+1}^{(l)} R . \\
H_{k+1, k}^{(l)}=R P^{T} \\
\hat{H}_{k}^{(l)}=\left[\begin{array}{cc}
\hat{H}_{k-1}^{(l)} & F_{k}^{(l)} \\
0 & H_{k+1, k}^{(l)}
\end{array}\right] \\
U_{[k+1]}^{(l)}=\left[U_{[k]}^{(l)}, U_{k+1}^{(l)}\right] \\
\mathrm{END} \mathrm{FOR}
\end{gathered}
$$

END FOR

It is important to use a rank-revealing $Q R$ decomposition [10] for the computation of $U_{k+1}^{(l)}$; it not only helps reduce memory requirements it also guarantees that the algorithm returns a $\mathcal{U}_{k}$ which is an orthonormal basis of $\mathcal{K}_{k}(\mathcal{A}, \mathcal{B})$. Note that all coefficients necessary to define the reduced Lyapunov equation (8) are computed by the algorithm. Setting

$$
H_{k}^{(l)}=\left[\begin{array}{ll}
\hat{H}_{k-1}^{(l)} & F_{k}^{(l)}
\end{array}\right]
$$

it can be shown that

$$
\begin{aligned}
\mathcal{U}_{k}^{T} \mathcal{A U}_{k} & =\left[\begin{array}{cccc}
0 & & & H_{k}^{(p)} \\
H_{k}^{(1)} & \ddots & & \\
& \ddots & \ddots & \\
& & H_{k}^{(p-1)} & 0
\end{array}\right]=: \mathcal{H}_{k} \\
\mathcal{U}_{k}^{T} \mathcal{B} & =\operatorname{diag}\left(R^{(1)}, \ldots, R^{(p)}\right)=: \mathcal{R},
\end{aligned}
$$

where the upper triangular matrices $R^{(l)}$ are computed in the first loop of Algorithm 3.

The reduced Lyapunov equation

$$
\mathcal{H}_{k}^{T} \mathcal{Y} \mathcal{H}_{k}-\mathcal{Y}=-\mathcal{R} \mathcal{R}^{T}
$$

has again a block diagonal solution $\mathcal{Y}=\operatorname{diag}\left(Y^{(1)}, \ldots, Y^{(p)}\right)$. Furthermore, equation (8) is equivalent to

$$
H_{k}^{(l) T} Y^{(l+1)} H_{k}^{(l)}-Y^{(l)}=-R^{(l)} R^{(l) T} .
$$

However, there is a fundamental difference between the original periodic Lyapunov equation (1) and its reduced counterpart (11). While all matrices $A^{(l)}$ are $n \times n$ the coefficient matrices of (11) may have time-varying dimensions, i.e., $H_{k}^{(l)} \in$ $\mathbb{R}^{n_{l} \times n_{l+1}}$, where $1 \leq n_{l} \leq n$ and $n_{1}=n_{p+1}$. There are some preprocessing steps necessary so that (11) can be solved with existent algorithms [22]. Note that even the assumption that the original coefficient matrices $A^{(l)}$ have constant dimensions could be omitted. 


\section{Application to Balanced Truncation}

We now show how the solution of periodic Lyapunov equations can be used to produce reduced order models of the linear discrete-time periodic system

$$
\begin{aligned}
x_{l+1} & =A^{(l)} x_{l}+B^{(l)} u_{l} \\
y_{l} & =C^{(l)} x_{l},
\end{aligned}
$$

where the matrices $A^{(l)} \in \mathbb{R}^{n \times n}, B^{(l)} \in \mathbb{R}^{n \times m}$ and $C^{(l)} \in$ $\mathbb{R}^{n \times m}$ are periodic with period $p$. Moreover, it is assumed that the system (12) is asymptotically stable, i.e.,

$$
\rho\left(A^{(p)} A^{(p-1)} \cdots A^{(1)}\right)<1 .
$$

Intimately related to the system properties of (12) are the solutions of the two periodic Lyapunov equations

$$
\begin{aligned}
& A^{(l)} P^{(l)} A^{(l) T}-P^{(l+1)}=-B^{(l)} B^{(l) T}, \\
& A^{(l) T} Q^{(l+1)} A^{(l)}-Q^{(l)}=-C^{(l) T} C^{(l)} .
\end{aligned}
$$

The matrices $P^{(1)}, \ldots, P^{(p)}$ and $Q^{(1)}, \ldots, Q^{(p)}$ form the so called reachability and observability Gramians, respectively. Note that (13) is not a periodic Lyapunov equation in the strict sense of (1). However, the algorithms derived in Sections 2 and 3 can also be applied to (14) by an appropriate index change.

Now let $P^{(l)}=S^{(l) T} S^{(l)}$ and $Q^{(l)}=R^{(l) T} R^{(l)}$ be the Cholesky factorisations of the Gramians. In analogy to the case $p=1$ [20] it was shown in [23] how to use ordered singular value decompositions

$$
R^{(l)} S^{(l) T}=\left[U_{1}^{(l)}, U_{2}^{(l)}\right]\left[\begin{array}{cc}
\Sigma_{1}^{(l)} & 0 \\
0 & \Sigma_{2}^{(l)}
\end{array}\right]\left[V_{1}^{(l)}, V_{2}^{(l)}\right]^{T}
$$

$l=1, \ldots, p$, where $\Sigma_{1}^{(l)}, \Sigma_{2}^{(l)}$ are diagonal and the diagonal entries of $\Sigma_{1}^{(l)}$ are positive, to obtain a balanced truncated model of (12). From the decompositions above define the truncation matrices

$L^{(l)}=\left(\Sigma_{1}^{(l)}\right)^{-1 / 2} U_{1}^{(l) T} R^{(l)}, \quad T^{(l)}=S^{(l) T} V_{1}^{(l)}\left(\Sigma_{1}^{(l)}\right)^{-1 / 2}$

and let

$$
A_{r}^{(l)}=L^{(l+1)} A^{(l)} T^{(l)}, B_{r}^{(l)}=L^{(l+1)} B^{(l)}, C_{r}^{(l)}=C^{(l)} T^{(l)},
$$

where $L^{(p+1)}=L^{(1)}$. Then the reduced system is given by

$$
\begin{aligned}
\tilde{x}_{l+1} & =A_{r}^{(l)} \tilde{x}_{l}+B_{r}^{(l)} u_{l} \\
\tilde{y}_{l} & =C_{r}^{(l)} \tilde{x}_{l} .
\end{aligned}
$$

As in the previous section it is not necessary to insist on time constant dimensions, neither for the original system nor for the reduced system.

The truncation error of the reduction can be bounded as follows.
Theorem 4 [23] Let $G$ and $G_{r}$ be the transfer function matrices associated with (12) and (16), respectively. Then

$$
\left\|G(z)-G_{r}(z)\right\|_{\infty} \leq 2 \sum_{l=1}^{p} \operatorname{tr}\left(\Sigma_{2}^{(l)}\right)
$$

where $\Sigma_{2}^{(p)}$ is defined in (15).

It suggests itself to combine the Krylov subspace method derived in Section 3 with balanced truncation model reduction. This leads to a periodic variant of oblique projection methods for large scale model reduction [13].

\section{Application to Periodic Riccati Equations}

Consider the discrete-time periodic Riccati equation

$$
\begin{aligned}
0= & C^{(l) T} Q^{(l)} C^{(l)}-X^{(l)}+A^{(l) T} X^{(l+1)} A^{(l)} \\
- & A^{(l) T} X^{(l+1)} B^{(l+1)} . \\
& \left(R^{(l+1)}+B^{(l+1) T} X^{(l+1)} B^{(l+1)}\right)^{-1} . \\
& B^{(l+1) T} X^{(l+1)} A^{(l)},
\end{aligned}
$$

where the coefficient matrices are $A^{(l)} \in \mathbb{R}^{n \times n}, B^{(l)} \in$ $\mathbb{R}^{n \times m}, C^{(l)} \in \mathbb{R}^{n \times m}$ and $Q^{(l)} \in \mathbb{R}^{m \times m}, R^{(l)} \in \mathbb{R}^{m \times m}$ with $Q^{(l) T}=Q^{(l)} \geq 0, R^{(l) T}=R^{(l)}>0$. All matrices are assumed to be periodic with period $p$. Under certain assumptions [5], equation (17) has a unique solution $X^{(l)}$ with $X^{(l)}=X^{(l) T} \geq 0$. The periodic Riccati equation is an important tool to analyse and solve stabilization and linear-quadratic periodic optimal control problems [5, 19, 18].

Similar to the periodic Lyapunov equation, we can embed (17) in a standard Riccati equation,

$$
\begin{aligned}
0= & \mathcal{C}^{T} \mathcal{Q C}-\mathcal{X}+\mathcal{A}^{T} \mathcal{X} \mathcal{A} \\
& -\mathcal{A}^{T} \mathcal{X} \mathcal{B}\left(\mathcal{R}+\mathcal{B}^{T} \mathcal{X} \mathcal{B}\right)^{-1} \mathcal{B}^{T} \mathcal{X} \mathcal{A}
\end{aligned}
$$

where $\mathcal{A}$ is defined in (3) and $\mathcal{B}, \mathcal{C}, \mathcal{Q}, \mathcal{R}$ are block diagonal matrices with diagonal entries $B^{(l)}, C^{(l)}, Q^{(l)}, R^{(l)}, l=$ $1, \ldots, p$, respectively. If (18) has a unique positive semidefinite solution $\mathcal{X}$ then $\mathcal{X}$ is block diagonal with diagonal entries $X^{(1)}, \ldots, X^{(p)}$, which form a solution of (17) [5].

Applying Newton's method to (18) leads to the following iteration $[11,16]$.

\section{Algorithm 5}

$$
\begin{aligned}
\text { FOR } k= & 0,1,2, \ldots \\
\mathcal{K}_{k}= & \left(\mathcal{R}+\mathcal{B}^{T} \mathcal{X}_{k} \mathcal{B}\right)^{-1} \mathcal{B}^{T} \mathcal{X}_{k} \mathcal{A} \\
\mathcal{A}_{k}= & \mathcal{A}-\mathcal{B} \mathcal{K}_{k} \\
\mathcal{R}_{k}= & \mathcal{C}^{T} \mathcal{Q C}-\mathcal{X}_{k}+\mathcal{A}^{T} \mathcal{X}_{k} \mathcal{A} \\
& -\mathcal{A}^{T} \mathcal{X}_{k} \mathcal{B}\left(\mathcal{R}+\mathcal{B}^{T} \mathcal{X}_{k} \mathcal{B}\right)^{-1} \mathcal{B}^{T} \mathcal{X}_{k} \mathcal{A}
\end{aligned}
$$

Compute the solution $\mathcal{N}_{k}$ of the periodic Lyapunov equation

$$
\mathcal{A}_{k}^{T} \mathcal{N}_{k} \mathcal{A}_{k}-\mathcal{N}_{k}=-\mathcal{R}_{k} .
$$




$$
\begin{aligned}
& \quad \mathcal{X}_{k+1}=\mathcal{X}_{k}+\mathcal{N}_{k} \\
& \text { END FOR }
\end{aligned}
$$

It is easy to check that if $\mathcal{X}_{0}$ is block diagonal then the iterates $\mathcal{X}_{1}, \mathcal{X}_{2}, \ldots$ stay block diagonal. Thus, Algorithm 5 can be rewritten in terms of the coefficient matrices. The derivation of the corresponding algorithm is straightforward and therefore omitted. The following conditions $[11,16]$ guarantee that the iterates $\mathcal{X}_{k}$ converge globally quadratic to $\mathcal{X}$, the solution of (18):

1. $(\mathcal{A}, \mathcal{B})$ is stabilisable,

2. there exists a unique stabilising solution $\mathcal{X}$ so that $\mathcal{R}+$ $\mathcal{B}^{T} \mathcal{X} \mathcal{B}$ is positive definite,

3. $X_{0}$ is stabilising.

While 1 and 2 are standard control theoretic assumptions it is a major flaw of Algorithm 5 that it requires a stabilising initial guess $\mathcal{X}_{0}$. Also, a poor initial guess may lead to a large error $\left\|\mathcal{X}-\mathcal{X}_{0}\right\|$ which may imply that the initial convergence is very slow. Since the periodic Lyapunov equation (19) must be solved in each iteration it is important to achieve rapid convergence.

For these and other reasons, Newton's method should only be used as a defect correction method or for iterative refinement of an approximate solution obtained by a different algorithm. Following [2] we propose to use Algorithm 5 in a hybrid setting. First, a structure-preserving but potentially unstable algorithm [8] is used to compute a stabilising initial guess $\mathcal{X}_{0}$. Then, Algorithm 5 is used to refine this solution. From the experiences with $p=1$ it can be expected that usually no more than 2 Newton iterations are necessary to obtain an approximate solution of (17) with sufficiently small residual [2].

\section{Conclusions}

We have presented new variants of the squared Smith iteration and Krylov subspace based methods for the approximate solution of discrete-time periodic Lyapunov equations. It must be mentioned that these methods have not been implemented yet and numerical results are still to come. However, the known results for the case $p=1$ may indicate that our new algorithms provide a viable alternative to existing algorithms when the coefficients matrices become large and sparse. An implementation of the oblique projection method outlined at the end of Section 3 is currently under progress. At this time, this seems to be the most promising method for model reduction of discretetime periodic systems.

\section{Acknowledgments}

The author thanks Prof. V. Mehrmann and Dr. V. Sima for useful comments on an earlier version of this paper. This work was supported by the DFG Research Center "Mathematics for Key Technologies" (FZT 86) in Berlin.

\section{References}

[1] A.C. Antoulas, Sorensen D.C., and Zhou Y. On the decay rate of the Hankel singular values and related issues. Sys. Control Lett., 46(5):323-342, 2002.

[2] P. Benner and H. Faßbender. A hybrid method for the numerical solution of discrete-time algebraic Riccati equations. Contemporary Mathematics, 280:255-269, 2001.

[3] P. Benner, E.S. Quintana-Ortí, and G. Quintana-Ortí. Numerical solution of discrete stable linear matrix equations on multicomputers. Parallel Algorithms and Applications, 17(1):127-146, 2002.

[4] S. Bittanti, P. Colaneri, and G. De Nicolao. Two techniques for the solution of the discrete-time periodic Riccati equation. In Linear algebra in signals, systems, and control (Boston, MA, 1986), pages 315-331. SIAM, Philadelphia, PA, 1988.

[5] S. Bittanti, P. Colaneri, and G. De Nicolao. The periodic Riccati equation. In S. Bittanti, A. J. Laub, and J. C. Willems, editors, The Riccati Equation, pages 127-162. Springer-Verlag, Berlin, Heidelberg, Germany, 1991.

[6] P. Bolzern and P. Colaneri. The periodic Lyapunov equation. SIAM J. Matrix Anal. Appl., 9(4):499-512, 1988.

[7] R. Byers and N. Rhee. Cyclic Schur and HessenbergSchur numerical methods for solving periodic Lyapunov and Sylvester equations. Technical report, Dept. of Mathematics, Univ. of Missouri at Kansas City, 1995.

[8] W. R. Ferng, W.-W. Lin, and C.-S. Wang. On computing the stable deflating subspaces of cyclic symplectic matrix pairs. submitted for publication, cited in [14].

[9] F.R. Gantmacher. The Theory of Matrices. Chelsea, New York, 1960.

[10] G. H. Golub and C. F. Van Loan. Matrix Computations. Johns Hopkins University Press, Baltimore, MD, third edition, 1996.

[11] G.A. Hewer. An iterative technique for the computation of steady state gains for the discrete optimal regulator. IEEE Trans. Automat. Control, AC-16:382-384, 1971.

[12] I.M. Jaimoukha and E.M. Kasenally. Krylov subspace methods for solving large Lyapunov equations. SIAM J. Numer. Anal., 31:227-251, 1994.

[13] I.M. Jaimoukha and E.M. Kasenally. Oblique projection methods for large scale model reduction. SIAM J. Matrix Anal. Appl., 16(2):602-627, 1995.

[14] W.-W. Lin, P. Van Dooren, and Xu Q.-F. Periodic invariant subspaces in control. In Proc. of IFAC Workshop on Periodic Control Systems, Como, Italy, 2001. 
[15] S. Longhi and G. Orlando. Balanced reduction of linear periodic systems. Kybernetika (Prague), 35(6):737-751, 1999. 6th IEEE Mediterranean Conference on Control and Systems (Alghero, 1998).

[16] V. Mehrmann. The Autonomous Linear Quadratic Control Problem, Theory and Numerical Solution. Number 163 in Lecture Notes in Control and Information Sciences. Springer-Verlag, Heidelberg, July 1991.

[17] R. A. Smith. Matrix equation $X A+B X=C$. SIAM J. Appl. Math., 16:198-201, 1968.

[18] J. Sreedhar and P. Van Dooren. On finding stabilizing state feedback gains for a discrete-time periodic system. In American Control Conf., pages 1167-1168, 1994.

[19] J. Sreedhar and P. Van Dooren. A Schur approach for solving some periodic matrix equations. In U. Helmke, R. Mennicken, and J. Saurer, editors, Systems and Networks : Mathematical Theory and Applications, volume 77, pages 339-362. Akademie Verlag, Berlin, 1994.

[20] M.S. Tombs and I. Postlethwaite. Truncated balanced realization of a stable non-minimal state-space system. Internat. J. Control, 46(4):1319-1330, 1987.

[21] A. Varga. Periodic Lyapunov equations: some applications and new algorithms. Internat. J. Control, 67(1):6987, 1997.

[22] A. Varga. Balancing related methods for minimal realization of periodic systems. Systems Control Lett., 36(5):339-349, 1999.

[23] A. Varga. Balanced truncation model reduction of periodic systems. In Proc. of IEEE Conference on Decision and Control, CDC'2000, Sydney, Australia, 2000.

[24] A. Varga and S. Pieters. Gradient-based approach to solve optimal periodic output feedback control problems. $A u$ tomatica, 45:477-481, 1998. 